\title{
Statin-induced immune-mediated necrotising myopathy: a case report
}

CCD Leung, MBChB, MRCP (UK); WY Chu, FHKCP, FHKAM; CF Ko, FHKCP, FHKAM

\begin{abstract}
Immune-mediated necrotising myopathy is a rare disorder characterised by muscle necrosis, fatigue, and weakness. We report one such case with symptom onset more than 3 years after regular statin use. This report serves as a diagnostic alert when a patient's statin-associated muscle symptoms fail to resolve a few weeks after statin discontinuation.
\end{abstract}

Key words: Atorvastatin; Autoantibodies; Hydroxymethylglutaryl-CoA reductase inhibitors; Muscle weakness
Department of Medicine \& Geriatrics North Lantau Hospital, Tung Chung, Hong Kong

Correspondence to: CCD Leung, Department of Medicine $\mathcal{E}$ Geriatrics North Lantau Hospital, Tung Chung, Hong Kong. Email: lcc487@ha.org.hk

\section{CASE PRESENTATION}

In April 2020, a 79-year-old man with type-2 diabetes mellitus and hyperlipidaemia presented with a 2-month history of progressive muscle weakness and tea-coloured urine. His usual medications included gliclazide (160 mg twice daily), dapagliflozin (10 mg daily), and metformin (1 g twice daily). The patient was also taking statins for persistently elevated low-density lipoprotein level of $3.8 \mathrm{mmol} / \mathrm{L}$ : simvastatin (20 mg every night) since January 2016, which changed to atorvastatin (40 mg every night) in July 2018. He denied using over-thecounter drugs or traditional Chinese medicine. On admission, the patient's Glasgow Coma Scale was 15/15. Neurological examination revealed upper limb power of $4 / 5$ and lower limb power of $3 / 5$, with predominantly proximal muscle weakness. His sensations, reflexes, cranial examination results were normal, and he had no obvious muscle wasting.

Blood test results revealed raised levels of creatine kinase to $9019 \mathrm{U} / \mathrm{L}$ (reference range, 69-272 U/L) and alanine transaminase to $312 \mathrm{IU} / \mathrm{L}$ (reference range, $<50 \mathrm{IU} / \mathrm{L}$ ). His blood levels of sodium, potassium, calcium, haemoglobin, white cell count, and platelet were all within the normal range. Urine myoglobin was positive. Anti-nuclear antibodies, antiENA, anti-Jo-1, myositis-specific antibody panel including anti-signal recognition particle antibody were negative. Anti-3-hydroxy-3-methylglutaryl coenzyme A reductase (HMGCR) antibody was not checked because the test was not available in public service.

A diagnosis of statin-related rhabdomyolysis was made. Atorvastatin was stopped immediately, and intravenous fluid was given. The patient's creatine kinase level went down but remained persistently high at around 3000-6000 U/L. 3 weeks later, his muscle weakness further deteriorated to $3 / 5$ in the upper limbs and 2/5 in the lower limbs. In May 2020, the neurology team was consulted, and a muscle biopsy was taken for suspected immune-mediated necrotising myopathy (IMNM). Empirical high dose prednisolone (20 mg twice daily) was started. His creatine kinase level eventually decreased to $829 \mathrm{U} / \mathrm{L}$, but his limb power further deteriorated to $2 / 5$ on all four limbs. In June 2020, the patient acquired hospital-acquired pneumonia and died. In July 2020, the biopsy report confirmed the diagnosis of IMNM.

\section{DISCUSSION}

Statin therapy is associated with a variety of neuromuscular disorders, including inflammatory myopathies such as polymyositis, dermatomyositis, and IMNM, which is a rare disease with a prevalence of 7 to 11 cases per 100000 people in the United States. ${ }^{1}$ The European Neuromuscular Centre classifies IMNM into three subtypes: recognise anti- 
signal recognition particle myopathy, anti-HMGCR myopathy, and autoantibody-negative IMNM. ${ }^{1}$

Patients with IMNM typically have symmetric proximal muscle weakness progressing over weeks to months. The neck flexor, pharyngeal, and respiratory muscles may also be involved, ${ }^{2}$ which may predispose patients to pneumonia. Rarely, extra-muscular involvement such as rash, arthritis, or interstitial lung disease may be present. ${ }^{3}$ This disease is defined by muscle fibre necrosis on biopsy and the presence of autoantibodies to signal recognition particle or HMGCR, which is the pharmacologic target of statin medications. Statin use is reported to precede the development of anti-HMG-CoA reductase myopathy in $11 \%$ to $100 \%$ of cases. ${ }^{4}$

Histologically, IMNM is characterised by scattered necrotic and regenerating fibres (often more regenerating fibres than fibres undergoing necrosis), with relative absence of lymphocyte infiltration and abundance of macrophage infiltration. ${ }^{5}$ Unlike patients with self-limited statinassociated muscle symptoms, patients taking statins who are anti-HMGCR positive seldom improve after discontinuation of statins. Rather, these patients develop a progressive myopathic process that requires immunosuppressive therapy to control. ${ }^{3}$

There are no clinical trials to guide IMNM treatment. The treatment decisions are derived from the personal experience of clinicians, case series, and observational studies. The European Neuromuscular Centre recommends corticosteroids plus methotrexate as initial treatment. ${ }^{1}$ Alternatives to methotrexate include azathioprine, mycophenolate, tacrolimus, cyclosporine, or cyclophosphamide. Intravenous immunoglobulin may be used in refractory disease. A combination of treatments including high-dose corticosteroids, methotrexate, rituximab, and intravenous immunoglobulin may be required to achieve a clinical response in the most severely affected individuals.

Muscle prognosis is worse in IMNM than most other classes of myositis, and the percentage of patients with IMNM who have persistent weakness despite intense immunosuppressant treatment is large. This explains the deterioration of muscle power in our patient despite biochemical improvement in creatine kinase level after starting high-dose prednisolone.
In conclusion, IMNM should be suspected whenever statin-associated muscle symptoms fail to resolve several weeks after statin discontinuation. Test for anti-HMGCR antibody may have led to an earlier diagnosis, but it is unavailable in our public hospital.

\section{CONTRIBUTORS}

All authors designed the study, acquired the data, analysed the data, drafted the manuscript, and critically revised the manuscript for important intellectual content. All authors had full access to the data, contributed to the study, approved the final version for publication, and take responsibility for its accuracy and integrity.

\section{CONFLICTS OF INTEREST}

All authors have disclosed no conflicts of interest.

\section{FUNDING/SUPPORT}

This study received no specific grant from any funding agency in the public, commercial, or notfor-profit sectors.

\section{DATA AVAILABILITY}

All data generated or analysed during the present study are available from the corresponding author on reasonable request.

\section{ETHICS APPROVAL}

The patient was treated in accordance with the tenets of the Declaration of Helsinki. The patient's wife provided written informed consent for all treatments and procedures and for publication.

\section{REFERENCES}

1. Pinal-Fernandez I, Casal-Dominguez M, Mammen AL. Immune-mediated necrotizing myopathy. Curr Rheumatol Rep 2018;20:21. Crossref

2. Watanabe $\mathrm{Y}$, Uruha A, Suzuki S, et al. Clinical features and prognosis in anti-SRP and anti-HMGCR necrotising myopathy. J Neurol Neurosurg Psychiatry 2016;87:1038-44. Crossref

3. Mammen AL. Autoimmune myopathies. Continuum (Minneap Minn) 2016;22:1852-70. Crossref

4. GeY, Lu X, Peng Q, Shu X, Wang G. Clinical characteristics of anti3-hydroxy-3-methylglutaryl coenzyme A reductase antibodies in Chinese patients with idiopathic inflammatory myopathies. PLoS One 2015;10:e0141616. Crossref

5. Chung T, Christopher-Stine L, Paik JJ, Corse A, Mammen AL. The composition of cellular infiltrates in anti-HMG-CoA reductaseassociated myopathy. Muscle Nerve 2015;52:189-95. Crossref 